\title{
BMJ Open Gambling and the COVID-19 pandemic in the province of Quebec (Canada): protocol for a mixed-methods study
}

\author{
Magaly Brodeur (D) , ${ }^{1}$ Sophie Audette-Chapdelaine, ${ }^{1}$ Annie-Claude Savard, ${ }^{2}$ \\ Sylvia Kairouz ${ }^{3}$
}

To cite: Brodeur M, AudetteChapdelaine S, Savard A-C, et al. Gambling and the COVID-19 pandemic in the province of Quebec (Canada): protocol for a mixedmethods study. BMJ Open 2021;11:e048785. doi:10.1136/ bmjopen-2021-048785

- Prepublication history for this paper is available online. To view these files, please visit the journal online (http://dx.doi. org/10.1136/bmjopen-2021048785).

Received 06 January 2021 Accepted 25 August 2021

Check for updates

(C) Author(s) (or their employer(s)) 2021. Re-use permitted under CC BY-NC. No commercial re-use. See rights and permissions. Published by BMJ.

'Département de médecine de famille et de médecine d'urgence, Université de Sherbrooke, Sherbrooke, Quebec, Canada

2École de travail social et de criminologie, Université Laval, Quebec, Quebec City, Canada ${ }^{3}$ Department of Sociology and Anthropology, Concordia University, Montreal, Québec, Canada

Correspondence to Dr Magaly Brodeur; magaly.brodeur@usherbrooke. ca

\section{ABSTRACT}

Introduction The COVID-19 pandemic has major collateral impacts on mental health. Gambling is among the major public health issues that seems to have been transformed by the pandemic. In the province of Quebec in Canada, gambling is an important leisure activity. About two out of three adults are in Quebec gamble. The objective of this study is to draw a portrait of the impacts of the COVID-19 pandemic on gamblers and to learn more about their experiences during the pandemic in the province of Quebec.

Method and analysis This study has a sequential explanatory mixed-method design in two phases. The first phase is a cross-sectional online survey with Quebec residents who are 18 years of age or older and have gambled at least once in the previous 12 months. The second phase will be a qualitative study. Semistructured interviews will be conducted with gamblers, family members, addiction counsellors and state representatives selected through purposing sampling.

Ethics and dissemination This study is one of the first mixed-methods studies on the impacts of the COVID-19 pandemic on gambling. This study will generate new scientific knowledge on a worrisome public health issue, that is, gambling, and provide a better understanding of the experiences and gambling behaviours of gamblers during the pandemic. This study is funded by the Ministry of Health and Social Services of the Government of Quebec and was approved on 27 October 2020 by the Scientific and Research Ethics Committee of the CIUSSS de l'EstrieCHUS. This is a 2-year study that will be completed in June 2022.

\section{INTRODUCTION}

The COVID-19 pandemic is having a major impact on our daily lives. To help prevent the spread of the virus, health authorities have enacted various measures to protect citizens and mitigate the progression of the pandemic (physical distancing, lockdown, etc). These public health measures are having major collateral impacts, particularly on mental health, which has quickly become a concern for the scientific community and policy-makers. ${ }^{1-5}$ An increase in anxiety and depressive symptoms has been observed in

\section{Strengths and limitations of this study}

This is one of the first mixed-methods studies on the impacts of the COVID-19 pandemic on gambling

- The results will provide a more comprehensive view of gambler's experience during the pandemic.

- Gamblers were involved in the development of the questionnaire and will be involved in the implementation and the dissemination of the study.

- The quantitative phase is a cross-sectional survey and the sampling is non-probabilistic.

- Participants must be 18 years of age or older, reside in the province of Quebec and have gambled at least once in the previous 12 months to be included in the study.

many countries ${ }^{4}$ and similar observations have also been made about the use of potentially addictive products (alcohol, cannabis, online gaming, use of pornography, etc).$^{6-9}$

Many concerns have also been raised about gambling, which is an important public health issue. Several stakeholders are concerned about the consequences of the pandemic on gambling (eg, shift to online gambling because of the closure of physical gambling spaces and increase in gambling problems among individuals suffering from concomitant disorders, such as depression and anxiety). ${ }^{1011}$ The situation is concerning because, in the past, it has been shown that gambling tends to increase during the crises. ${ }^{12-14}$

In the province of Quebec in Canada, gambling is an important leisure activity. In 2018 , about two out of three adults $(65.6 \%)$ engaged in at least one form of gambling, and nearly one-third of the Quebec population (29.5\%) reported gambling at least once a month up to once a week. ${ }^{15}$ In March 2020, to enforce public health regulations, the government of Quebec temporarily suspended several gambling activities (casinos, lotteries, etc) to address the pandemic. This suspension 
of services has had major impacts. Gamblers' assistance services became overwhelmed with requests for help, and several actors were concerned about the resurgence of online gambling that seems to be occurring in the province. $^{16-18}$

Since the beginning of the pandemic, a few studies have been published on the impacts of the COVID-19 pandemic on gambling. ${ }^{10}{ }^{19-27}$ These studies are mainly quantitative and focused on gambling habits during the pandemic. In this context, it is possible to notice a lack of qualitative and mixed-method literature on the subject and realise that we know very little about gamblers' experiences during the pandemic. The objective of this study is to fill these gaps.

\section{METHODS AND ANALYSIS}

Aim

The objective of this study is to draw a portrait of the impacts of the pandemic on gamblers and to learn more about the experiences of gamblers during the pandemic in the province of Quebec.

\section{Design}

This study uses a sequential explanatory mixed research design. ${ }^{28} \mathrm{~A}$ mixed sequential and explanatory research design is a two-phase project that begins with the collection and analysis of quantitative data (phase I), followed by the collection and analysis of qualitative data (phase II). The objective of phase II is to enrich and explain the information obtained in phase I. 'The overall intent of this design is to have the qualitative data help explain in more detail the initial quantitative results'. ${ }^{28}$ The questions in the qualitative phase will be based on the information obtained in the quantitative phase.
The use of a mixed-methods design combines the strength of quantitative and qualitative methods. ${ }^{29}$ These methods are frequently used in research on complex issues such as mental disorders and gambling. ${ }^{30-32}$ The study design is presented in figure 1 .

\section{Eligibility criteria}

Participants must be 18 years of age or older, reside in the province of Quebec and have gambled at least once in the previous 12 months to be included in the study. This information is collected through a self-reported questionnaire at the beginning of the study. Participants must speak and understand French to participate in the study.

\section{Phase I: quantitative study}

Phase I involves a cross-sectional online survey in French. The objective is to learn more about the impacts of the COVID-19 pandemic on gamblers and to draw a portrait of their gambling habits.

\section{Sample size and sampling method}

The sample size will be 1250 gamblers, which was determined based on the existing literature on gambling. ${ }^{32-37}$ The sampling method will be non-probabilistic, and participants will be recruited online voluntarily using social media.

\section{Data collection}

The survey is divided into four sections: (1) demographics; (2) general impacts of COVID-19 (social relationships, family life, mental health); (3) impacts of COVID-19 on gambling and (4) health profile and experience of healthcare.

The first section on the demographic characteristics of participants includes questions on age, gender, first

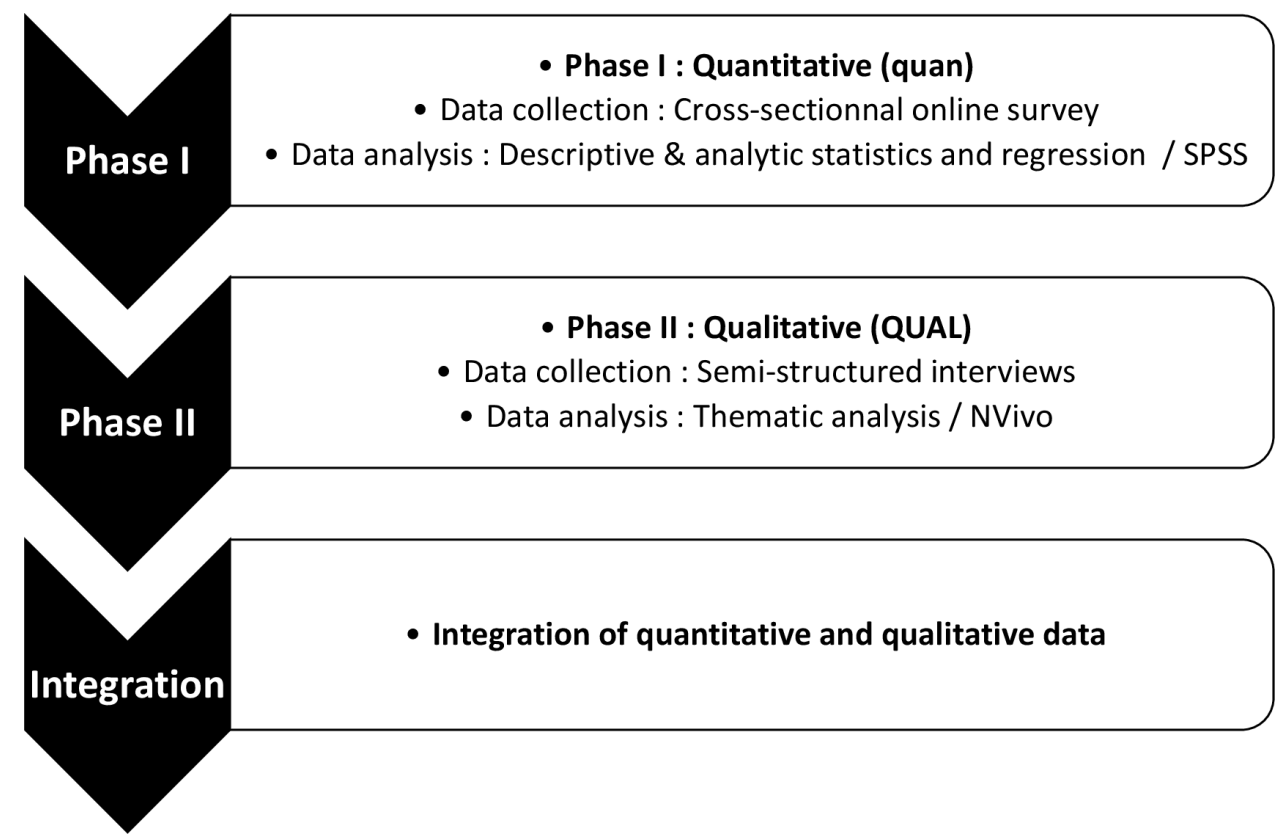

Figure 1 Study design visual. 
language, ethnic origin, region of residence, marital status, family status, employment, annual income and level of education. To determine the impact of COVID-19 on the sociodemographic profiles of the participants, we will also examine the impact of the pandemic on marital status, employment, and annual income by asking them about their situation before and after the beginning of the pandemic.

The second section includes questions on the general impacts of COVID-19 (social relationships, family life and mental health). This section was inspired by the Statistics Canada survey on the impacts of COVID-19. It includes questions on the impact of COVID-19 on lifestyle habits (sleep, nutrition and diet, tobacco, alcohol, drugs, cannabis, use of pornography, physical activity and social activities). The mental health status of each participant will be evaluated by administering the Patient Health Questionnaire-4 (PHQ-4).

The PHQ-4 is a validated screening scale used to screen for general anxiety and depressive symptoms. ${ }^{38}$ The PHQ-4 consists of an anxiety subscale (Generalized Anxiety Disorder-2 (GAD-2)) and a depression subscale (PHQ-2), with two items for each. The four items are added up to produce the PHQ-4 total score. A four-point Likert scale ranging from $0=$ not at all to $3=$ nearly every day is used to rate the items. The PHQ-4 total score is used to classify the symptoms of anxiety and depression as follows: none (0-2), mild (3-5), moderate (6-8) and severe (9-12). This tool is validated in French. ${ }^{39} 40$

The third section includes questions on the impacts of the COVID-19 pandemic on gambling behaviours (frequency of gambling, length of gambling sessions and amount of money spent before and since the beginning of the pandemic). It also includes an assessment of problem gambling through the administration of the Problem Gambling Severity Index (PGSI). ${ }^{41}$

The PGSI is a nine-item validated instrument designed to measure problem gambling in the general population. For each item, respondents answer using a four-point scale from $0=$ never to $3=$ almost always. The PGSI total score is used to classify gambling as follows: non-problem gambler (0); low-risk gambler (1-2), moderate-risk gambler (3-7) and problem gambler (8 and over). This tool is validated in French. ${ }^{42}$

The fourth section aims to develop a health profile of the participants and learn more about their comorbidities (anxiety, depression, substance use, etc) and their experiences of healthcare and services during the pandemic.

\section{Data analysis}

Quantitative data will be analysed with SPSS and SAS software. Descriptive statistics will be generated to describe our sample. Categorical variables will be presented with frequencies and percentages. Normally distributed continuous variables will be presented with the mean and SD or with the median and IQRs (IQR: 25-75th percentiles).
The PGSI score will be categorised into four levels. Sociodemographic variables, PHQ-4 and gamblingrelated variables will be compared across the four levels of the PGSI using $\chi^{2}$ for categorical variables or the exact Monte Carlo method if there are too small frequencies. Continuous variables will be compared with analysis of variance or Kruskal-Wallis test depending on whether the data are normally distributed or not, respectively.

A multivariate logistic regression will be performed to evaluate variables that could explain a score PGSI $\geq 3$ or $<3$. Variables with $\mathrm{p}<0.1$ in the univariate analyses will be included in the multivariate model. A stepwise selection will be performed to keep only the variables with a $p<0.05$ in the final model.

As there are many variables analysed, a false discovery rate correction will be used to reduce the risk of error. After adjustment, a $\mathrm{p}<0.05$ will be considered significant.

\section{Phase II: qualitative study}

Phase II includes semistructured interviews with gamblers. Complementary interviews will also be conducted with the gamblers' families, addiction counsellors, Loto-Québec representatives and policy-makers from the Ministry of Health and Social Services of the government of Quebec.

\section{Sample size and sampling method}

Twenty-five gamblers will be selected by purposing sampling among the phase I participants who agree to be contacted for phase II of the study. Gamblers will be selected to ensure a diversity of profiles based on the results of the quantitative phase. Certain criteria (age, gender, comorbidity, etc) will be used to select participants to ensure maximum variability. Sampling will continue until data saturation is reached.

Participants for the complementary interviews (ministry of health and social services (1), Loto-Québec (1), addiction counsellors (2), gambler's families (3)) will also be selected by purposing sampling in collaboration with gambling: help and referral, the ministry of health and social services and Loto-Québec.

The information provided by these various sources will allow us to better understand the political, administrative and social context related to gambling in Quebec during the pandemic.

\section{Data collection}

Data will be collected through online or in-person semistructured interviews, depending on public health restrictions. The two main topics covered during the interviews will be the participant's experiences during the COVID-19 pandemic and the impacts of the pandemic on their health and gambling habits.

The interviews with family members, addiction workers, Loto-Québec representatives and decision-makers from the Ministry of Health and Social Services will help us learn more about the policy-making process, the issues related to access to care for gamblers, and the impacts of the pandemic on gamblers. We will also ask all actors 
what they believe are the best courses of action to take to improve healthcare and services for gamblers.

The interviews will be recorded and transcribed verbatim. Data collection will continue until saturation is reached for gamblers and no new themes emerge in the interviews.

\section{Data analysis}

Data will be imported into NVivo software to carry out a thematic analysis. Thematic analysis is a methodology used to systematically identifying, organising, coding and offering insight into a data set by the creation of themes. ${ }^{43}$

The verbatim of the interviews will first be coded and classified by themes, independently, by two members of the research team. The initial codes will be examined for similarities and differences during consensus meetings. These themes will then be organised into similar groups to create a thematic tree structure. The team will then organise codes into a hierarchy to create final themes. Iterative phases of analysis will be carried out. These iterative phases will allow the team members to take ownership of the content of the analysis and the coding process, but will also help the team to develop a common understanding of the context and the data being studied. This iterative and reflexive process will allow the triangulation of team members' expertise (medical, psychological, social work, etc) and knowledge.

\section{Interpretation of quantitative and qualitative data}

After completing the two phases, the quantitative and qualitative data will be interpreted in concert with each other, as expected in a mixed design, ${ }^{28}$ in order to obtain a better understanding of the experiences of gamblers during the pandemic and the impacts of the crisis on their health and gambling habits.

\section{Public involvement statement}

A gambler was involved in the development of the questionnaire and will be involved in the implementation and the dissemination of the study. Gamblers were not involved in the development of the original design of this study.

\section{Ethics and dissemination}

This study has been approved by the Scientific and Research Ethics Committee of the Integrated University Health and Social Services Centre of the Estrie regionUniversity Hospital Center of Sherbrooke (CIUSSS de l'Estrie-CHUS) under the number \#2021-3818. Electronic and/or written informed consent, depending on the data collection format (online survey and online or in-person interviews), will be obtained from each participant. A copy of the consent form and contact information will be delivered to each participant.

This research is funded by the Ministry of Health and Social Services of the Government of Quebec in collaboration with the Quebec Research Fund and the Ministry of Innovation and Economy as part of the COVID-19 call for solutions (funding number \#20-CP-00309). The
Quebec Research Fund is a recognised and an open access advocating research-funding body listed by the JULIET project.

The results of this study will be shared with the Ministry of Health and Social Services of the Government of Quebec, Loto-Quebec, gambling: help and referral, researchers and the general public, and will be submitted for publication.

\section{CONCLUSION}

This study is, to our knowledge, one of the first mixedmethods studies on the impacts of the COVID-19 pandemic on gamblers. ${ }^{44} 45$ This study will generate new scientific knowledge on a major public health issue, that is, gambling, and provide a better understanding of the experiences of gamblers during the pandemic. This study has some limitations. First, the quantitative phase is a cross-sectional survey. A longitudinal survey would have provided a better understanding and a more complete description of the impact of the pandemic on the gamblers. Second, the sampling is non-probabilistic and the results cannot be generalised. Third, this study only concerns gamblers aged 18 and over.

Despite these limitations, this study will fill an important gap in the literature as there are only few mixed studies on the subject. ${ }^{44}$ The use of a mixed design will provide a more comprehensive picture of gamblers' experiences during the pandemic and better knowledge about their gambling habits. This study will also allow us to learn more about the general mental health status of gamblers during the pandemic. Obtaining these data is crucial for the development of public policies related to gambling to help this population during not only the remainder of the pandemic but also future crises.

This 2-year study will end in June 2022.

Acknowledgements We would like to thank Annie Desjardins, patient-partner, for her involvement in the study and the revision of the protocol. We would also like to thank Gambling: help and referral for their collaboration on the project.

Contributors MB designed and wrote the original proposal. All authors participated in discussing the design of the study and developing the research protocol. MB was responsible for drafting this manuscript and was supported by SA-C, A-CS and SK. All authors read and approved the final manuscript. All authors are responsible for data collection and analysis.

Funding This research is funded by the Ministry of Health and Social Services of the Government of Quebec in collaboration with the Quebec Research Fund and the Ministry of Innovation and Economy as part of the COVID-19 call for solutions (funding number \#20-CP-00309).

Disclaimer The funding body is not involved in the research and the researchers are independent.

Competing interests None declared.

Patient and public involvement Patients and/or the public were involved in the design, or conduct, or reporting, or dissemination plans of this research. Refer to the Methods section for further details.

Patient consent for publication Not required.

Provenance and peer review Not commissioned; externally peer reviewed.

Open access This is an open access article distributed in accordance with the Creative Commons Attribution Non Commercial (CC BY-NC 4.0) license, which permits others to distribute, remix, adapt, build upon this work non-commercially, 
and license their derivative works on different terms, provided the original work is properly cited, appropriate credit is given, any changes made indicated, and the use is non-commercial. See: http://creativecommons.org/licenses/by-nc/4.0/.

ORCID iD

Magaly Brodeur http://orcid.org/0000-0003-3856-1877

\section{REFERENCES}

1 Galea S, Merchant RM, Lurie N. The mental health consequences of COVID-19 and physical distancing. JAMA Intern Med 2020;180:817.

2 Holmes EA, O'Connor RC, Perry VH, et al. Multidisciplinary research priorities for the COVID-19 pandemic: a call for action for mental health science. Lancet Psychiatry 2020;7:547-60.

3 Pfefferbaum B, North CS. Mental health and the Covid-19 pandemic. N Engl J Med Overseas Ed 2020;383:510-2.

4 Rajkumar RP. COVID-19 and mental health: a review of the existing literature. Asian J Psychiatr 2020;52:102066.

5 Torales J, O'Higgins M, Castaldelli-Maia JM, et al. The outbreak of COVID-19 coronavirus and its impact on global mental health. Int J Soc Psychiatry 2020;66:317-20.

6 Koopmann A, Georgiadou E, Kiefer F, et al. Did the general population in Germany drink more alcohol during the COVID-19 pandemic Lockdown? Alcohol Alcohol 2020;55:698-9.

7 Bartel SJ, Sherry SB, Stewart SH. Self-isolation: a significant contributor to cannabis use during the COVID-19 pandemic. Subst Abus 2020;41:1-4.

8 King DL, Delfabbro PH, Billieux J, et al. Problematic online gaming and the COVID-19 pandemic. J Behav Addict 2020;9:184-6.

9 Mestre-Bach G, Blycker GR, Potenza MN. Pornography use in the setting of the COVID-19 pandemic. J Behav Addict 2020;9:181-3.

10 Håkansson A, Fernández-Aranda F, Menchón JM, et al. Gambling during the COVID-19 crisis - a cause for concern?. J Addict Med 2020;14:e10-12.

11 Yahya AS, Khawaja S. Problem gambling during the COVID-19 pandemic. Prim Care Companion Cns Disord 2020;22.

12 Economou M, Souliotis K, Malliori M, et al. Problem gambling in Greece: prevalence and risk factors during the financial crisis. $J$ Gambl Stud 2019;35:1193-210.

13 Jiménez-Murcia S, Fernández-Aranda F, Granero R, et al. Gambling in Spain: update on experience, research and policy. Addiction 2014;109:1595-601.

14 Olason DT, Hayer T, Brosowski T, et al. Gambling in the mist of economic crisis: results from three national prevalence studies from Iceland. J Gamb/ Stud 2015;31:759-74

15 du QG. Les jeux de hasard et d'argent au Québec et en régions: Statistiques de participation en 20182018.

16 Loto-Québec. Fermeture de la vente des loteries CheZ les détaillants et dans les kiosques, 2020. Available: https://societe.lotoquebec. com/fr/covid-19/vente-detaillants-suspendues

17 QMI A. Loto-Québec débordée par les appels. Journal de Montréal 2020.

18 Radio-Canada. La fermeture des casinos peut-elle avoir nui aux joueurs excessifs? | Radio-Canada.ca, 2020. Available: https:// ici.radio-canada.ca/nouvelle/1719973/jeu-compulsif-excessifpathologique-dependance-casino-coronavirus-loto-quebec

19 Auer M, Malischnig D, Griffiths MD. Gambling before and during the COVID-19 pandemic among European regular sports Bettors: an empirical study using behavioral tracking data. Int J Ment Health Addict 2020:1-8.

20 Griffiths S, Reith G, Wardle H, et al. Pandemics and epidemics: public health and gambling harms. Public Health 2020;184:1-2.

21 Håkansson A. Changes in gambling behavior during the COVID-19 pandemic - a web survey study in Sweden. Int J Environ Res Public Health 2020;17:4013.

22 Håkansson A. Impact of COVID-19 on online gambling - a general population survey during the pandemic. Front Psychol 2020;11:568543.

23 Johnstone P, Regan M. Gambling harm is everybody's business: a public health approach and call to action. Public Health 2020;184:63-6.
24 Lindner P, Forsström D, Jonsson J, et al. Transitioning between online gambling modalities and decrease in total gambling activity, but no indication of increase in problematic online gambling intensity during the first phase of the COVID-19 outbreak in Sweden: a time series forecast study. Front Public Health 2020;8:554542.

25 Håkansson A, Jönsson $C$, Kenttä G. Psychological distress and problem gambling in elite athletes during COVID-19 Restrictions-A web survey in top Leagues of three sports during the pandemic. Int $J$ Environ Res Public Health 2020;17:6693.

26 Price A. Online gambling in the midst of COVID-19: a nexus of mental health concerns, substance use and financial stress. Int $J$ Ment Health Addict 2020:1-18.

27 Sharman S. Gambling in football: how much is too much? Managing Sport Leis 2020:1-8.

28 Creswell JW, Creswell DJ. Research design: qualitative, quantitative, and mixed methods approaches. Sage Edition. Thousand Oaks: Sage publications, 2018

29 Pluye P, Hong QN. Combining the power of stories and the power of numbers: mixed methods research and mixed studies reviews. Annu Rev Public Health 2014;35:29-45.

30 Dobbs PD, Hodges EJ, Dunlap CM, et al. Addiction vs. dependence: a mixed methods analysis of young adult JUUL users. Addict Behav 2020;107:106402.

31 Seaman EL, Howard DE, Green KM, et al. A sequential explanatory mixed methods study of young adult tobacco and marijuana Co-Use. Subst Use Misuse 2019;54:2177-90.

32 John B, Holloway K, Davies N, et al. Gambling harm as a global public health concern: a mixed method investigation of trends in Wales. Front Public Health 2020;8:320.

33 Sirola A, Kaakinen M, Oksanen A. Excessive gambling and online gambling communities. J Gamb/ Stud 2018;34:1313-25.

34 Griffiths MD. The use of online methodologies in data collection for gambling and gaming addictions. Int J Ment Health Addict 2010;8:8-20.

35 Lorains FK, Cowlishaw S, Thomas SA. Prevalence of comorbid disorders in problem and pathological gambling: systematic review and meta-analysis of population surveys. Addiction 2011;106:490-8.

36 Cowlishaw S, Gale L, Gregory A, et al. Gambling problems among patients in primary care: a cross-sectional study of general practices. Br J Gen Pract 2017;67:e274-9.

37 Pearson C, Hussain Z. Gaming and technology addiction: breakthroughs in research and practice. Pennsylvania: IGI Global, 2017: 212-29.

38 Kroenke K, Spitzer RL, Williams JBW, et al. An ultra-brief screening scale for anxiety and depression: the PHQ-4. Psychosomatics 2009;50:613-21.

39 Arthurs E, Steele RJ, Hudson M, et al. Are scores on English and French versions of the PHQ-9 comparable? an assessment of differential item functioning. PLoS One 2012;7:e52028.

40 Micoulaud-Franchi J-A, Lagarde S, Barkate G, et al. Rapid detection of generalized anxiety disorder and major depression in epilepsy: validation of the GAD-7 as a complementary tool to the NDDI-E in a French sample. Epilepsy Behav 2016;57:211-6.

41 Holtgraves T. Evaluating the problem gambling severity index. $J$ Gambl Stud 2009;25:105-20.

42 McCready J, Adlaf E. Performance and enhancement of the Canadian problem gambling index (CPGl): report and recommendations, 2006. Available: https://prism.ucalgary.ca/handle/ $1880 / 44244$

43 Clarke V, Braun V. Thematic analysis. J Posit Psychology 2016;12:1-2.

44 Hunt K, Critchlow N, Brown A, et al. Protocol for a mixed-method investigation of the impact of the COVID-19 pandemic and gambling practices, experiences and marketing in the UK: the "Betting and Gaming COVID-19 Impact Study". Int J Environ Res Public Health 2020;17:8449.

45 Wardle H, Donnachie C, Critchlow N, Hunt N, et al. The impact of the initial Covid-19 lockdown upon regular sports bettors in Britain: findings from a cross-sectional online study. Addict Behav 2021;118:106876. 